QMW-91-24

\title{
TEMPERATURE DEPENDENCE OF STRING THEORY IN THE PRESENCE OF WORLD-SHEET BOUNDARIES
}

\author{
Michael B. Green, \\ Department of Physics, \\ Queen Mary and Westfield College, London, UK
}

\begin{abstract}
The effect of world-sheet boundaries on the temperature-dependence of bosonic string theory is studied to first order in string perturbation theory. The high- temperature behaviour of a theory with Dirichlet boundary conditions has features suggestive of the high-temperature limit of the confining phase of large- $n S U(n)$ Yang-Mills theory, recently discussed by Polchinski.
\end{abstract}


The question of whether there is an exact correspondence between QCD and some kind of interacting string theory is of long-standing interest. Although the quark and gluon picture of the structure of hadrons is simple and useful for discussing scattering at high energy and fixed angle or deep inelastic processes it is an awkward way of describing the bound states of the theory. The possibility of expressing QCD as some kind of string theory is motivated both by the lattice formulation of $S U(n)$ Yang-Mills theory as well as by the large $n$ limit in the continuum. Holding $g^{2} n$ fixed as $n \rightarrow \infty$ (where $g$ is the Yang-Mills coupling constant) leads to 't Hooft's topological expansion of Yang-Mills theory in a power series in $1 / n[1]$. The diagrams in this expansion correspond to the world-sheets of string theory where the closed-string coupling constant, $\kappa$, is related to the Yang-Mills coupling by $\kappa=\alpha g^{2}$ (where $\alpha$ is a constant) and is therefore inversely proportional to $n$. According to the folklore of the large- $n$ approximation glueball states may be associated with closed strings and meson states with open strings carrying quark flavour quantum numbers at their end-points, whereas baryons are very massive solitonic states [2] (about which nothing will be said in this paper).

Obviously a string theory of hadrons has to have rather different properties from a string theory of the 'conventional' type that describes gravity together with other forces. Firstly, the observed spectrum of hadrons does not include massless spin-1 and spin-2 gauge particles. Furthermore, fixed-angle scattering cross sections decrease exponentially with energy in conventional string theories whereas experimental hadronic cross sections decrease as a power of the energy at fixed angle — a crucial indication of point-like substructure. An added constraint is that hadrons must couple correctly to external (electro-weak) sources, which is a natural property of QCD but does not seem possible for string theories describing gravity.

In order to compare QCD with string theory Polchinski recently [3] considered the high temperature limit of the confining phase of large- $n S U(n)$ Yang-Mills theory (in this limit the system is unstable since there is a deconfining phase transition at a finite temperature). He discussed the correlation functions of Wilson loops that wrap $q$ times around the periodic imaginary time dimension (where the period $\beta$ is the inverse temperature) and are fixed at spatial points. The form of the one-loop free energy indicates that the dominant states that mediate these gauge-invariant correlation functions are tachyonic glueball states with masses 


$$
M_{Y M}^{2}=-\frac{2 g^{2} n}{\pi^{2} \beta^{2} q^{2}}
$$

as $\beta \rightarrow 0$ and to lowest order in perturbation theory.

The temperature-dependent spectrum of conventional tree- level string theory looks nothing like (1). The 'mass' spectrum of a closed-string theory with a compact euclidean time coordinate of circumference $\beta$ is given (at tree level) by

$$
\alpha^{\prime} M_{\text {string }}^{2} \equiv-\alpha^{\prime} \mathbf{p}^{2}-\frac{4 \pi^{2} \alpha^{\prime} m^{2}}{\beta^{2}}=\frac{\beta^{2} q^{2}}{4 \pi^{2} \alpha^{\prime}}+2 N+2 \tilde{N}-4
$$

where $\mathbf{p}$ denotes the spatial components of the momentum $p^{I}(I=1, \ldots, D)$ and $m$ is the integer Kaluza-Klein charge of the quantized $p^{0}$ (and $\alpha^{\prime}=1 / \pi T$, where $T$ is the string tension). At low temperature (large $\beta$ ) states of non-zero winding have positive $M_{\text {string. }}^{2}$. The Hagedorn transition at $\beta^{2}=16 \pi^{2} \alpha^{\prime}$ is seen to occur at the temperature at which the lowest-mass winding state $(m=N=\tilde{N}=0$ and $q=1)$ becomes massless [4-6]. If the expression for $M_{\text {string }}^{2}$ is continued to $\beta \sim 0$ it is quite dissimlar from (1) in its dependence on $\beta$. Accordingly, Polchinski suggested that a string theory of QCD would be one in which the effective number of world-sheet fields grows with temperature at high temperature (in which case the -4 on the right-hand side of (2) is replaced by a $\beta$-dependent term). This paper will indicate that the temperature dependence of string theory may be substantially affected by the presence of world-sheet boundaries and this may lead to an (apparently) different resolution of the discrepency between the high temperature mass spectra of Yang-Mills theory and string theory.

The fact that $M_{Y M}^{2}$ is proportional to $g^{2} n$ means that it is associated with the lowest-order (genus zero) diagrams in the topological expansion of the large- $n$ theory. It is usually assumed that such diagrams are related to the tree diagrams of any corresponding string theory since the string coupling constant $\kappa$ is proportional to $1 / n$. For example, the radiative corrections associated with a loop of closed string give corrections to the mass spectrum of order $\kappa^{2} \sim 1 / n^{2}$ (and in any case are not of the correct form to produce the Yang-Mills mass spectrum (1) at high temperature). However, more interesting corrections arise in presence of world- sheet boundaries. The insertion of a single boundary (which changes the genus of the surface by $1 / 2$ ) is proportional to $w \kappa$, where $w$ is a weight which may be associated with internal symmetry in the Chan-Paton 
manner. In the usual formulation of open string theory the coordinate $X^{\mu}(\sigma, \tau)$ satisfies Neumann boundary conditions, i.e., the normal derivative vanishes, $\left.\partial_{n} X\right|_{B}=0$. In this case the theory describes interacting open and closed strings, and the boundaries are identified with the trajectories of free open-string end-points. The original string picture of mesons associated quark quantum numbers of a $U(m)$ flavour group with the free endpoints so that a closed world-sheet boundary carries the flavour weight $w \equiv w_{N}=$ const. $\times m$. The value of the constant is fixed by imposing perturbative unitarity in open-string channels. A string theory defined by summing over Riemann surfaces of arbitrary topology with these boundary conditions, which will be called a 'Neumann' theory, describes interacting open and closed strings. An alternative choice of boundary conditions requires the tangential derivative of the coordinate to vanish at the boundary, $\left.\partial_{t} X^{\mu}\right|_{B}=0$. This constant Dirichlet condition leads to a 'Dirichlet' theory, in which each world-sheet boundary is fixed at a space-time point, $y_{B}^{\mu}$, thereby defining a set of space-time correlation functions for point-like closed strings. Summing over all topologies and integrating over the $y_{B}^{\mu}$,s gives a radically modified Minkowskispace purely closed-string theory ([7,8] and references therein). The constant Dirichlet conditions result in power-behaved fixed-angle scattering amplitudes. The weight of a Dirichlet boundary may be naturally associated with the colour group, in which case $w \equiv w_{D}=$ const. $\times n$. In that case $w_{D} \kappa \sim g^{2} n$ and the boundary insertion contributes at the same order as the tree diagrams, even though it is of different genus.

The normalization of $w_{D}$, including its sign, is not fixed by conventional unitarity arguments. It is conceivable that it may be determined by a more subtle 'dual' form of unitarity based on the fact that the Dirichlet theory can be related to the Neumann theory by a spatial duality transformation in which all dimensions are compactified on circles of radius $R$ in the limit $R \rightarrow 0$ [7]. The $y_{B}^{\mu}$ enter the compactified Neumann theory as $U(1)$ gauge fields that are constant on each boundary. They may take different values on different boundaries in which case they must be integrated over their allowed range. [Alternatively the $y_{B}^{\mu}$ can be considered to be boundary values of world sheet scalar fields $Y^{\mu}(\sigma, \tau)$ (satisfying $\left.\partial_{t} Y^{\mu}\right|_{B}=0$ ) that couple to $\hat{X}^{\mu}$ (where $\hat{X}$ denotes a coordinate satisfying Neumann conditions) by a total derivative term proportional to $\int d \sigma d \tau \epsilon^{\alpha \beta} \partial_{\alpha} \hat{X}^{\mu} \partial_{\beta} Y_{\mu}$ in the action].

Figure 1 illustrates three representations of the insertion of a single boundary in 
Figure 1. The insertion of a single boundary (indicated by thick lines) in a closed-string propagator. (a) A representation in which the world-sheet exhibits intermediate open strings. In the Dirichlet theory these have end-points that are fixed at $y^{\mu}$ in space-time, which is integrated over in the amplitude. (b) A representation that exhibits a closed-string tadpole. (c) In the light-cone gauge (in which $\tau=X^{+}$ and $\left.P^{+}(\sigma)=p^{+} / 2 \pi\right)$ the Dirichlet boundary (represented by a vertical slit) forces a finite fraction of the total $p^{+}$carried by the string to accumulate at a single spatial point at 'time' $\tau=X_{0}^{+}$.

a closed-string propagator. The first of these (fig 1(a)) shows the intermediate (group singlet) open strings that mix with the closed ones. In the case of the Neumann theory at zero temperature this is the familiar mixing of open-string theory that leads, for example, to a Higgs-type mechanism that gives a mass to the level-one antisymmetric tensor state [9-11]. In the case of the Dirichlet theory there are no physical intermediate open-string states (apart from the level-1 vector which gives rise to a divergence in the zero-temperature theory). Furthermore, the position of the boundary $y^{\mu}$ is to be integrated. Figure 1(b) represents the insertion as a closed-string tadpole coupling to the vacuum via either a Neumann or a Dirichlet boundary. The presence of a massless dilaton in the cylinder channel makes the diagram divergent as usual. In figure 1(c) the Dirichlet boundary is illustrated in the light-cone gauge. It imposes an instantaneous condition (at light-cone time $\tau=X_{0}^{+}$) that forces a finite fraction of the light-cone momentum, $p^{+}$, to accumulate at a single space-time point which is then integrated.

The difference between the temperature-dependence of the expressions for the boundary insertions in the Neumann and Dirichlet theories is quite striking. In the case of the Neumann theory there are infinite numbers of intermediate open-string states in fig 1(a) carrying the same momentum as the closed strings. If a dimension is compactified winding number is not conserved - the intermediate open string does not possess 
a winding number (the Neumann boundary in fig 1(b) can carry winding number into the vacuum). Thus, the inverse propagator for a Neumann open string at inverse temperature $\beta$ carrying momentum $p^{\mu} \equiv\left(p^{I}, 2 \pi m / \beta\right)$ is given by

$$
\Delta_{N}^{-1}=\alpha^{\prime} \mathbf{p}^{2}+\frac{4 \pi^{2} m^{2}}{\beta^{2}}+N_{j}-1
$$

where $N_{j}$ is the level of an open-string state with occupation numbers labelled by $j$ and the normal ordering constant -1 arises from 24 transverse dimensions (or it may be made up from world-sheet conformal matter and ghost fields).

The only physical open-string state of the zero-temperature Minkowski-space Dirichlet theory is an isolated level-1 $U(n)$-singlet vector. The winding number associated with a toroidal compactification is now conserved - the fixed end-point intermediate open string carries the winding number, $q$, of the closed strings to which it couples and the propagator insertion is diagonal in winding number. The closed-string momentum is not conserved if $y$ is fixed but integration over $y$ imposes its conservation and guarantees translation-invariance of the Dirichlet theory. The inverse propagator of an open string with end-points fixed at $y_{1}$ and $y_{2}$ and inverse temperature $\beta$ is given by [7]

$$
\Delta_{D}^{-1}=\frac{1}{4 \alpha^{\prime} \pi^{2}}\left(y_{2}-y_{1}+q \beta\right)^{2}+N_{j}-1
$$

The intermediate open-string state in fig 1 (a) has $y_{1}=y_{2}=y$ so that the propagator does not depend on the $y_{B}$ 's. However, the open-string wave function still depends on $y$ (i.e., there is a continuum of open-string states and momentum conservation follows upon integrating over $y$ ).

The matrix elements of the propagator insertion have the form

$$
\Pi_{r s}=\sum_{j} c_{r, j} \Delta_{o p e n} c_{s, j}
$$

where $\Delta_{\text {open }}$ represents either (3) or (4) $\left(\Delta_{N}\right.$ and $\Delta_{D}$, respectively). The coefficients $c_{r, j}$ (which are proportional to the open-string coupling constant, $g_{\text {open }}$ ) define the couplings between a closed-string state with occupation numbers labelled by $r$ and open-string states with occupation numbers $j$. They are functions of $\left(\beta, p^{I}, m, q\right)$ and include a group theory factor $\delta^{a b}(a, b=1, \ldots, n)$ with indices that are contracted by the propagator in 
(5), leading to an overall factor of $n$ in $\Pi_{r s}$. The shift in the closed-string masses is determined by diagonalizing $\Pi_{r s}$. An important qualitative difference between the temperature dependence of the Neumann and Dirichlet theories can be seen directly from the structure of (3) and (4) (with $y_{1}=y_{2}$ ). In the Neumann case the propagator $\Delta_{N}$ has an unremarkable dependence on $\beta$ in the limit $\beta \rightarrow 0$ (states with $m \neq 0$ become infinitely massive and decouple, leaving only $m=0$ states). However, if the expression for the Dirichlet propagator, $\Delta_{D}$, is continued to $q \beta<<2 \pi \sqrt{\alpha^{\prime}}$ (ignoring the Hagedorn transition at $\left.\beta=4 \pi \sqrt{\alpha^{\prime}}\right) \Pi_{r s}$ is dominated by the $N=1, m=0$ openstring states in (5). These open-string vector states of non-zero winding have couplings $c_{r, 1}$ to closed-string states labelled $r$. [The zero winding-number open-string state at this level is the vector Lagrange multiplier field that causes divergences in the zerotemperature amplitudes in perturbation theory [7].] To first order in $w_{D} \kappa$ this gives a shift proportional to $G_{r} w_{D} \kappa / q^{2} \beta^{2}$ in the (mass) ${ }^{2}$ of closed-string states (recalling that $\kappa$ is proportional to $g_{\text {open }}^{2}$ ). The coefficient $G_{r}$ depends on precise details of the couplings, $c_{r, 1}$, that will be discussed below, but is a smooth non-vanishing function of $\beta$ for small $\beta$. Therefore, when continued to higher temperatures, $\beta^{2}<<\alpha^{\prime} G_{r} w_{D} \kappa / q^{2}$ the shifted mass (at first order in $w_{D} \kappa$ ) may have the same general form as that in (1) if the weight of the boundary is chosen so that $w_{D} \sim n$ (as expected for a Chan-Paton factor associated with the colour $U(n))$. Such a comparison is purely formal in the absence of any estimate of higher-order contributions to both the QCD and the string expressions (which will not be considered in this paper).

Figure 2. The insertion of arbitrary numbers of boundaries in an open-string propagator leads to higher-order mass corrections. 
The complete modification to the bare closed-string propagator due to boundary insertions, illustrated by fig 2 , involves mass insertions of arbitrarily high order in $w \kappa$ as well as being non-diagonal in the closed-string states. However, to lowest order in $w \kappa$ a single boundary insertion dominates $\Pi_{r s}$ and only those matrix elements that couple closed-string states of equal bare mass contribute. These perturbative mass shifts are then determined by the on-shell amplitude for coupling the two closed-string states, $r$ and $s$, to a disk with appropriate boundary conditions. We shall first use this to consider the simplest case which arises when $r$ and $s$ are the (non-degenerate) closed-string tachyonic ground-states with non-zero winding number and momentum satisfying (from hereon the tension will be chosen so that $\alpha^{\prime}=1$ for convenience)

$$
M_{\text {tachyon }}^{2}=-\mathbf{p}^{2}-\frac{4 \pi^{2} m^{2}}{\beta^{2}}=\frac{q^{2} \beta^{2}}{4 \pi^{2}}-4 .
$$

The two-tachyon amplitudes may be evaluated in the standard manner (or extracted from eq.(14) of [7]). With Neumann conditions at the boundary of the disk the amplitude with two ground states is

$$
\begin{aligned}
\Pi_{N} & =w_{N} \kappa \int_{0}^{1} \frac{d x}{x^{2}}(1-x)^{\frac{2 \pi^{2} m^{2}}{\beta^{2}}-\frac{q^{2} \beta^{2}}{8 \pi^{2}}+\frac{\mathrm{p}^{2}}{2}} \\
& =w_{N} \kappa \int_{0}^{1} \frac{d x}{x^{2}}(1-x)^{2-\frac{q^{2} \beta^{2}}{4 \pi^{2}}}
\end{aligned}
$$

where the mass-shell condition (6) has been used and an overall constant has been arbitrarily absorbed into the coupling since the normalizatiotion of the boundary insertion is arbitrary at this stage. The $x=0$ boundary of moduli space (where the two vertex operators coincide) gives rise to singularities associated with the emission of a closed-string tachyon and dilaton into the vacuum with zero momentum. These familiar divergences do not affect the dominant $\beta$ dependence at small $\beta$. The boundary $x=1$ (the region in which one of the vertex operators on the disk collides with the boundary if the other is held fixed at the centre of the disk) gives singularities at $\beta^{2}=4 \pi^{2}(3+N) / q^{2}$ (integer $N$ ). These are the values of $\beta$ at which the intermediate open- string states are on shell and at which the mass corrections are singular (the expression for $\Delta_{N},(3)$, has poles at these positions when (6) is used). In the limit $\beta \rightarrow 0$ (which is non-singular for finite $q$ ) the amplitude is constant. 
With Dirichlet conditions the two-tachyon disk amplitude becomes

$$
\begin{aligned}
\Pi_{D} & =\lim _{k \rightarrow 0} w_{D} \kappa \int_{0}^{1} \frac{d x}{x^{2}} x^{\frac{k^{2}}{4}}(1-x)^{-\frac{2 \pi^{2} m}{\beta^{2}}+\frac{q^{2} \beta^{2}}{8 \pi^{2}}-\frac{\mathbf{p}^{2}}{2}} \\
& =\lim _{k \rightarrow 0} w_{D} \kappa \int_{0}^{1} \frac{d x}{x^{2}} x^{\frac{k^{2}}{4}}(1-x)^{-2+\frac{q^{2} \beta^{2}}{4 \pi^{2}}} \\
& =\lim _{k \rightarrow 0} w_{D} \kappa \frac{\Gamma\left(-1+\frac{q^{2} \beta^{2}}{4 \pi^{2}}\right) \Gamma\left(-1+\frac{k^{2}}{4}\right)}{\Gamma\left(-2+\frac{q^{2} \beta^{2}}{4 \pi^{2}}+\frac{k^{2}}{4}\right)}
\end{aligned}
$$

where $k^{\mu}=-p^{1 \mu}-p^{2 \mu}$ is the momentum that leaks through the Dirichlet boundary but is set to zero by the $y^{\mu}$ integration (and $p^{\mu} \equiv p^{1 \mu}$ ). Again there is a $x=0$ singularity due to the emission of a dilaton at zero momentum (the Dirichlet condition avoids the zero-momentum tachyon divergence by defining $k^{2}=0$ by analytic continuation from $k^{2}>4$ ), which does not affect the dominant $\beta$ dependence at small $\beta$. The $x=1$ boundary gives rise to singularities at $q^{2} \beta / 4 \pi^{2}=1-N$ (which are the positions of the poles of $\Delta_{D}$ given by (4)) that are contained in the factor $\Gamma\left(-1+q^{2} \beta^{2} / 4 \pi^{2}\right)$. For $q \beta<<2 \pi$ the mass shift behaves as $8 \pi^{2} w_{D} \kappa / q^{2} \beta^{2}$ in accord with the earlier intuition. Furthermore, since the modified closed-string propagator has the form $\left(p^{2}+m^{2}-\Pi\right)^{-1} \sim$ $\left(p^{2}+m^{2}\right)^{-1} \Pi\left(p^{2}+m^{2}\right)^{-1}+\ldots$ the mass correction is negative if $w_{D}$ is chosen to have positive sign. The above argument assumed the shift is small relative to the tachyon mass, i.e., $4>>8 \pi^{2}\left|w_{D}\right| \kappa / q^{2} \beta^{2}$. If the result is nevertheless continued to values of $\beta \sim 0$ so that $M_{\text {tachyon }}^{2}+\delta M_{\text {tachyon }}^{2} \sim \delta M_{\text {tachyon }}^{2}$ the leading-order tachyon spectrum formally resembles that of (1), as was anticipated earlier. Tree-level corrections to the tachyon mass of higher order in $w \kappa$ arise from the insertion of more boundaries in the disk (for example, the diagram with two boundaries is an annulus that can be described as a loop of open string).

We now turn to consider the shift in the masses of arbitrary closed-string states to leading order in $w_{D} \kappa$. These are determined by the operator, $\Pi$, that inserts a single Dirichlet boundary in the world-sheet. The propagator joining two arbitrary closedstring states, $\langle X|$ and $|Y\rangle$, of momenta $p^{\mu} \equiv\left(p^{I}, m\right)$ and winding number $q$, can be formally expressed as a sum,

$$
\begin{aligned}
G(X, Y) & =\left\langle X\left|\Delta_{C}+\Delta_{C} \Pi \Delta_{C}+\ldots\right| Y\right\rangle \\
& =\left\langle X\left|\Delta_{C}\left(1-\Pi \Delta_{C}\right)^{-1}\right| Y\right\rangle
\end{aligned}
$$


where the bare closed-string propagator, $\Delta_{C}$, is given by $\Delta_{C}^{-1}=p^{2}+\beta^{2} q^{2} / 4 \pi^{2}+2 N+$ $2 \tilde{N}-4$ with $N=\tilde{N}$ (and $p^{2}=\mathbf{p}^{2}+4 \pi^{2} m^{2} / \beta^{2}$ ). The boundary insertion operator, $\Pi$, has the form

$$
\Pi=w_{D} \kappa \Upsilon^{\dagger} \Delta_{D} \Upsilon
$$

where $\Upsilon$ is the operator that couples a closed string to an open string with fixed endpoints (in the winding-number $q$ sector) and $\Delta_{D}$ is given by (4).

For $q \beta<<2 \pi, \Pi$ is dominated by the pole of $\Delta_{D}$ due to the $N=1$ open-string vector state with winding-number $q$. This state couples to a Dirichlet boundary via the interaction $\Upsilon \sim p^{\mu}|B\rangle^{\star}$, where $|B\rangle$ is the boundary state defined to satisfy the Dirichlet condition, $\partial_{\sigma} X^{\mu}(\sigma)|B\rangle=0$. The contribution to $\Pi$ of this state is then given by

$$
\Pi=K|B\rangle\langle B|
$$

where $K=2 \pi^{2} w_{D} \kappa p^{2} / q^{2} \beta^{2}$. It therefore contributes to the shift in mass of all the scalar closed- string states. For example, consider the finite set of equal-mass Fock-space endstates (labelled $r$ and $s$ ) at a given level with bare mass $\left(M_{r}^{2}=M_{s}^{2}=4 N_{r}-4+\beta^{2} q^{2} / 4 \pi^{2}\right)$. The shift in the position of the poles of (9) can be estimated to first order in $w \kappa$ by considering momenta such that $p^{2} \sim-M_{r}^{2}$ in (9). Substituting this and (11) into (9) gives

$$
\begin{aligned}
G(r, s) & \sim \frac{1}{p^{2}+M_{r}^{2}}\left[\delta_{r s}+\frac{K B_{r} B_{s}}{p^{2}+M_{r}^{2}-K B^{2}}\right] \\
& \sim \frac{1}{p^{2}+M_{r}^{2}}\left(\delta_{r s}-\frac{B_{r} B_{s}}{B^{2}}\right)+\frac{1}{p^{2}+M_{r}^{2}-K B^{2}}\left(\frac{B_{r} B_{s}}{B^{2}}\right)+\text { non-pole terms }
\end{aligned}
$$

where $B_{r}=\langle r \mid B\rangle, B^{2} \equiv \sum_{r} B_{r} B_{r}$ and $K B^{2}$ has been assumed to be small. If the level is non-degenerate (i.e., $r$ labels a single state) the first term in the second line of (12) (the 'transverse' term) vanishes and the the pole position is shifted to $M_{r}^{2}+\delta M_{r}^{2}$, where $\delta M_{r}^{2}=-K B^{2} \sim 2 \pi^{2} w_{D} \kappa M_{r}^{2} / q^{2} \beta^{2}$ (where the substitution $p^{2}=-M_{r}^{2}$ has been made in $K$, which is valid for calculations to lowest order in $\left.w_{D} \kappa\right)$. More generally, the levels are highly degenerate. In such cases only one combination of states in (12) (the

$\star$ This is the coupling between the isolated physical open-string state and the closed-string states that couple to the boundary. More generally, unphysical string states contribute to non- leading effects. 
'longitudinal one', $\left.|s\rangle B_{r} B_{s} / B^{2}\right)$ is shifted - again $\delta M_{r}^{2}=-K B^{2}$. The remaining states in (12) remain unshifted. If $w_{D}$ is chosen to be positive the shifts $\delta M_{r}^{2}$ are positive for states of positive $M_{r}^{2}$, the massless state (the dilaton) remains massless and the tachyon develops a more negative value of (mass) ${ }^{2}$ of the form determined earlier. If $w_{D}$ is negative the signs of the shifts are reversed and the possible correspondence with (1) is lost.

The tachyon state of winding number $q=1$ that plays an important rôle in determining the leading small $\beta$ behaviour when $w_{D}>0$ is precisely the state invoked in [4-6] as the origin of the Hagedorn transition at $\beta=4 \pi$. This is not a physical state of the zero temperature theory. An analogous unphysical tachyon plays a similar rôle in inducing the Hagedorn transition in superstring theory, where there is no zero-temperature tachyon state (and therefore the theory has a stable vacuum) [6]. A more consistent Dirichlet string theory than the one considered in this paper based on the ordinary bosonic theory (perhaps some version of the superstring theory of [12]) may be free of physical tachyons but might still display the $\beta \rightarrow 0$ behaviour characteristic of large- $n$ Yang- Mills theory.

In summary, this paper has considered the effect of the presence of world-sheet boundaries on the temperature dependence of bosonic string theories at high temperature. In general, this leads to a shift in the masses of states of non-zero winding number $(q \geq 1)$. With Dirichlet boundary conditions this effect can be particulary significant at high temperature since to first order in $w_{D} \kappa$ the mass shifts behave as $1 / \beta^{2}$ for $q \beta<<2 \pi$. Formally (i.e., ignoring the potentially important effects of terms of higher order in perturbation theory), the $\beta \rightarrow 0$ limit of the theory with $w_{D}>0$ has a tachyonic spectrum analogous to that of large- $n U(n)$ Yang-Mills theory discussed by Polchinski [3]. Any such connection requires the weight, $w_{D}$, associated with a Dirichlet boundary to be proportional to $n$ so that that the boundary insertions contribute at the same order as the usual string tree diagrams even though they are of higher genus. The precise relevance of this connection is difficult to assess, particularly since the comparison is between the continuation to high temperature of the low-temperature phases of the two theories the existence of the deconfining phase transition of Yang-Mills theory and the Hagedorn transition of string theory signifies that these phases are unstable and the spectrum is tachyonic. Furthermore, this comparison may be radically affected by higher-order terms that contribute to both theories. Nevertheless, together with the fact that the Dirichlet theory possesses power-behaved fixed-angle scattering amplitudes, this could 
be circumstantial evidence of a correspondence between Yang-Mills theory and some more realistic version of Dirichlet string theory.

The $q \neq 0$ terms (terms of non-zero winding number) that dominate the $\beta \rightarrow 0$ limit are infinitely suppressed as $\beta \rightarrow \infty$. The dominant $(q=0)$ term of the zerotemperature theory has an apparently problematic divergence due to the presence of the $N=1$ open-string vector intermediate state. This acts like a Lagrange multiplier field [7] which generates a significant shift of the bare closed-string spectrum which remains to be elucidated. Such a shift indicates the inadequacy of string perturbation theory the tree diagrams are substantially modified even for small $w_{D} \kappa$. Arguments based on [7] suggest that Dirichlet boundaries might lead to a modification to the usual gauge invariance so that the perturbative graviton, open-string vector and dilaton fields $\left(h^{\mu \nu}\right.$, $A^{\mu}$ and $\left.\phi\right)$ transform (in linearized approximation) as

$$
\delta h^{\mu \nu}=\partial^{(\mu} \xi^{\nu)}, \quad \delta A^{a b \mu}=g \xi^{\mu} \delta^{a b}+\partial^{\mu} \Lambda^{a b}, \quad \delta \phi=g \Lambda_{a}^{a},
$$

plus terms that vanish in the $\alpha^{\prime} \rightarrow 0$ limit. These transformations are reminiscent of the transformations of Stückelberg fields that enter into the gauge-invariant formulation of the Pauli-Villars action for a massive spin-two field of mass $\sim w_{D} \kappa$. The absence of a massless spin-two state would be most welcome if the theory is of relevance to hadronic physics but calculations of low order diagrams have not so far revealed the existence of such a mass term explicitly.

All the considerations in this paper are based on standard perturbation theory rules for critical string theory (in which any number of spatial dimensions can be replaced by conformal fields in a more or less standard manner). It may be that these ideas have more direct relevence in the context of string theory in sub-critical dimensions. In any case the presence of important boundary effects in Dirichlet string theory that modify tree-level amplitudes suggests that the standard determination of the critical dimension is likely to be significantly modified. 


\section{REFERENCES}

1. G. 't Hooft, A planar diagram theory for strong interactions, Nucl. Phys. B72 (1974) 461.

2. E. Witten, Baryons in the $1 / N$ expansion, Nucl. Phys. B160 (1979) 57.

3. J. Polchinski, High temperature limit of the confining phase, Austin preprint UTTG-26-91.

4. B. Sathapalian, Vortices on the string world-sheet and constraints on toral compactification, Phys. Rev. D35 (1987) 3277.

5. Ya.I. Kogan, Vortices on the world- sheet of a string; critical dynamics, JETP Lett. 45 (1987) 709.

6. J. Atick and E. Witten, The Hagedorn transition and the number of degrees of freedom of string theory, Nucl. Phys. B310 (1988) 291.

7. M.B. Green, Space-time duality and Dirichlet string theory, Phys. Lett. 266B (1991) 325.

8. M.B. Green, Point-like structure in string theory, Invited talk at the first international Sakharov conference, Moscow, June 1991 (QMW-91-11, to be published).

9. E. Cremmer and J. Scherk, Spontaneous dynamical breaking of gauge symmetry in dual models, Nucl. Phys. B72 (1974) 117.

10. M. Kalb and P. Ramond, Classical direct interstring action, Phys. Rev. D9 (1974) 2273.

11. J. Shapiro and C.B. Thorn,BRST-invariant transitions between closed and open strings, Phys. Rev. D36 (1987) 432.

12. M.B. Green, Wilson loops for strings and superstrings at finite temperature, QMW91-19. 